\title{
A NOTE ON FUNCTIONAL A POSTERIORI ESTIMATES FOR ELLIPTIC OPTIMAL CONTROL PROBLEMS
}

\author{
M. WOLFMAYR
}

\begin{abstract}
In this work, new theoretical results on functional type a posteriori estimates for elliptic optimal control problems with control constraints are presented. More precisely, we derive new, sharp, guaranteed and fully computable lower bounds for the cost functional in addition to the already existing upper bounds. Using both, the lower and the upper bounds, we arrive at two-sided estimates for the cost functional. We prove that these bounds finally lead to sharp, guaranteed and fully computable upper estimates for the discretization error in the state and the control of the optimal control problem. First numerical tests are presented confirming the efficiency of the a posteriori estimates derived.
\end{abstract}

\section{INTRODUCTION}

During the last couple of decades, the optimization of systems governed by partial differential equations (PDEs) has become more and more important in research and application, for which Lions has definitely paved the way with his work 23] in 1971. Books considering PDE-constrained optimization are, for instance, Hinze et al. [13, Tröltzsch [38, Borzì and Schulz 4] and Leugering et al. 22, 21]. Besides the PDE-constraints, the optimization problems often include control constraints given through a non-empty, convex and closed subset of a Hilbert space. In many cases, the set of admissible controls is represented in terms of inequality constraints (or box constraints) imposed on the controls.

A posteriori error estimates and adaptive methods for elliptic optimal control problems are the topic of many works, see, e.g., [2, 3, 24, 1, 9, 10, 11, 39, 14, which are mainly on residual-type a posteriori error estimates. Regarding a posteriori error estimates for control constrained optimal control problems, we also refer to the recent works [15, 16]. There are different approaches to a posteriori error estimation. Besides the residual-type estimates, there is also the class of functional type a posteriori error estimates. These techniques were introduced by S. Repin in the 90's, see, e.g., 30, 31, 32, 34, 33, 27. Later the functional type a posteriori estimates were also considered and obtained for optimal control problems, see [7, 8] as well as the books [35, 25] and the references therein. The benefit of functional type a posteriori estimates is that - as the name indicates - they are only derived by functional methods. Therefore, these estimates do not depend on the mesh and provide guaranteed upper bounds for the discretization errors.

In [7, sharp, guaranteed and fully computable upper bounds (majorants) for cost functionals of distributed elliptic optimal control problems were already presented. Now, we want to complete the functional type a posteriori error analysis of distributed elliptic optimal control problems by the derivation of guaranteed and fully computable lower bounds (minorants) for the cost functionals. The optimization problems of this work also include control constraints. The presented minorants are not only fully computable but sharp. Moreover, we prove that the new minorants and the already obtained majorants of [7] can be used in order to derive functional type a posteriori error estimates for the discretization error in the state and the control. The properties of the majorants and minorants are transferred to the majorants for the discretization error, i.e., they are guaranteed, fully computable and sharp.

The paper is organized as follows: In Section 2, we discuss the optimal control problem and its optimality system as well as present the already known results on majorants for the cost functional. Then, Section 3 is devoted to the derivation of new, fully computable minorants for the cost functional. Both results, the older ones on majorants as well as the new ones on minorants, together lead to guaranteed and fully computable upper bounds for the discretization 
error in the state and the control. These majorants are presented in Section 4. They attain their exact lower bound on the exact solution of the optimal control problem and, hence, are sharp. In Section 5. we present the corresponding results for the optimal control problem in the case without any inequality constraints imposed on the control. Section 6 is devoted to the finite element discretization of the optimal control problem and its iterative solution method in order to derive an approximation of the solution. We present first numerical results in Section 7 and finally draw some conclusions in Section 8 .

\section{The Elliptic Optimal Control Problem}

Let $\Omega \subset \mathbb{R}^{d}, d \in\{1,2,3\}$, be a bounded Lipschitz domain with boundary $\Gamma:=\partial \Omega$, and, let us denote the state of our optimal control problem by $y$ and the control by $u$. Given $y_{d} \in L^{2}(\Omega)$, $u_{d} \in L^{2}(\Omega), f \in L^{2}(\Omega)$ and $\lambda \in \mathbb{R}_{+}$, we consider the following distributed elliptic optimal control problem: Minimize the cost functional

$$
\mathcal{J}(y(v), v):=\frac{1}{2}\left\|y-y_{d}\right\|^{2}+\frac{\lambda}{2}\left\|v-u_{d}\right\|^{2}
$$

over $y \in V:=H_{0}^{1}(\Omega)$ and $v \in U_{\text {ad }} \subset L^{2}(\Omega)$ subject to the boundary value problem

$$
\begin{aligned}
-\operatorname{div}(\nu(\boldsymbol{x}) \nabla y(\boldsymbol{x})) & =f(\boldsymbol{x})+v(\boldsymbol{x}) & & \boldsymbol{x} \in \Omega, \\
y(\boldsymbol{x}) & =0 & & \boldsymbol{x} \in \Gamma .
\end{aligned}
$$

The set of admissible controls $U_{\text {ad }}$ is given by

$$
U_{\mathrm{ad}}=\left\{v \in L^{2}(\Omega): u_{a} \leq v \leq u_{b} \text { a.e. in } \Omega\right\},
$$

where $u_{a}, u_{b} \in L^{2}(\Omega)$ and $u_{a}(\boldsymbol{x}) \leq u_{b}(\boldsymbol{x})$ for almost all $\boldsymbol{x} \in \Omega$. The diffusion coefficient $\nu(\cdot)$ is assumed to be measurable, uniformly positive and bounded, i.e., satisfies the assumptions

$$
0<\underline{\nu} \leq \nu(\boldsymbol{x}) \leq \bar{\nu}, \quad \boldsymbol{x} \in \Omega
$$

where $\underline{\nu}$ and $\bar{\nu}$ are constants. The positive regularization parameter $\lambda$ provides a weighting of the cost of the control in the cost functional $\mathcal{J}(\cdot, \cdot)$. In these work, we denote by $(\cdot, \cdot)$ and $\|\cdot\|$ the inner products and norms in $L^{2}(\Omega)$, respectively, whereas the standard inner products and norms in $H^{1}(\Omega)$ are denoted by $(\cdot, \cdot)_{1}$ and $\|\cdot\|_{1}$, respectively.

Our goal is to approach to the desired state function $y_{d}$ and to the desired control $u_{d}$ as close as possible by finding a suitable control function $u$. Note that, in many problems, the desired control is given by $u_{d}=0$. The optimal control problem (1)-(3) has a unique solution (for the proof, see, e.g., [13, 38]) that can be also derived via the optimality conditions. Hence, the optimal solution of the optimality system is equivalent to the solution of the optimal control problem (11)-(3). In order to formulate the optimality system, we consider the following Lagrange functional for the minimization problem:

$$
\mathcal{L}(y(v), v, p(v)):=\mathcal{J}(y(v), v)+\int_{\Omega}(\operatorname{div}(\nu(\boldsymbol{x}) \nabla y(\boldsymbol{x}))+f(\boldsymbol{x})+v(\boldsymbol{x})) p(\boldsymbol{x}) d \boldsymbol{x},
$$

where $p$ denotes the Lagrange multiplier (adjoint state). The Lagrange functional (5) has to be understood in the weak sense. It has a saddle point, see, e.g., 13, 38. Hence, the corresponding (optimal) solution $(y, u, p) \in V \times U_{\text {ad }} \times V$ satisfies the system of (first order) necessary optimality conditions

$$
\begin{aligned}
\mathcal{L}_{p}(y(u), u, p(u)) & =0, \\
\mathcal{L}_{y}(y(u), u, p(u)) & =0, \\
\left(\mathcal{L}_{u}(y(u), u, p(u)), w-u\right)_{L^{2}(\Omega)} & \geq 0 \quad \forall w \in U_{\mathrm{ad}},
\end{aligned}
$$


which can be written in the weak form as follows:

$$
\begin{array}{rlrl}
\int_{\Omega} \nu \nabla y \cdot \nabla w d \boldsymbol{x} & =\int_{\Omega}(f+u) w d \boldsymbol{x} & & \forall w \in V, \\
\int_{\Omega} \nu \nabla p \cdot \nabla w d \boldsymbol{x} & =\int_{\Omega}\left(y-y_{d}\right) w d \boldsymbol{x} & & \forall w \in V, \\
\int_{\Omega}\left(p+\lambda\left(u-u_{d}\right)\right)(w-u) d \boldsymbol{x} & \geq 0 & \forall w \in U_{\mathrm{ad}},
\end{array}
$$

for (optimal) $y, p \in V$ and $u \in U_{\text {ad }}$.

Remark 1. In the unconstrained case, i.e., $U_{\mathrm{ad}}=L^{2}(\Omega)$, the variational inequality (8) of the optimality system simplifies to the equation $\mathcal{L}_{u}(y(u), u, p(u))=0$. Hence, the optimality condition (8) is then simplified to

$$
p+\lambda\left(u-u_{d}\right)=0 \quad \text { in } \Omega .
$$

Majorants for the cost functional. Guaranteed and fully computable upper bounds for the cost functional $\mathcal{J}$ have already been presented in [7], i.e.,

$$
\mathcal{J}(y(v), v) \leq \mathcal{J}^{\oplus}(\alpha, \beta ; \eta, \boldsymbol{\tau}, v) \quad \forall v \in U_{\mathrm{ad}}
$$

and for arbitrary $\alpha, \beta>0, \eta \in V$ and

$$
\boldsymbol{\tau} \in H(\operatorname{div}, \Omega):=\left\{\boldsymbol{\tau} \in\left[L^{2}(\Omega)\right]^{d}: \operatorname{div} \boldsymbol{\tau} \in L^{2}(\Omega)\right\} .
$$

Although the admissible set is different in [7] and the elliptic boundary value problem is stated without a diffusion parameter $\nu(\cdot)$, the majorants can be analogously derived for the optimal control problem (11)-(3). Including a diffusion parameter that meets the assumptions (4) in the model problem (2), the majorant $\mathcal{J}^{\oplus}$ is given by

$$
\begin{aligned}
\mathcal{J}^{\oplus}(\alpha, \beta ; \eta, \boldsymbol{\tau}, v):= & \frac{1+\alpha}{2}\left\|\eta-y_{d}\right\|^{2}+\frac{(1+\alpha)(1+\beta) C_{F}^{2}}{2 \alpha \underline{\nu}^{2}}\|\boldsymbol{\tau}-\nu \nabla \eta\|^{2} \\
& +\frac{(1+\alpha)(1+\beta) C_{F}^{4}}{2 \alpha \beta \underline{\nu}^{2}}\|f+v+\operatorname{div} \boldsymbol{\tau}\|^{2}+\frac{\lambda}{2}\left\|v-u_{d}\right\|^{2},
\end{aligned}
$$

where $C_{F}>0$ is the constant coming from the Friedrichs inequality. The parameters $\alpha, \beta>0$ have been introduced in order to obtain a quadratic functional by applying Young's inequality. The arbitrary functions $\eta \in V$ and $v \in U_{\text {ad }}$ can be taken as the approximate solutions of the optimal control problem (1)-(3) and $\tau \in H(\operatorname{div}, \Omega)$ represents the image of the exact flux $\nu \nabla \eta$. For the derivation of (11), the following estimate for the approximation error has been used:

$$
\|\nabla y(v)-\nabla \eta\| \leq \frac{1}{\underline{\nu}}\left(\|\boldsymbol{\tau}-\nu \nabla \eta\|+C_{F}\|f+v+\operatorname{div} \boldsymbol{\tau}\|\right) .
$$

The majorant (11) provides a sharp upper bound of the cost functional, if it is minimized over $\eta, \tau, v$ and $\alpha, \beta>0$, i.e.,

$$
\inf _{\substack{\eta \in V, \boldsymbol{\tau} \in H(\operatorname{div}, \Omega), v \in U_{\text {ad }}, \alpha, \beta>0}} \mathcal{J}^{\oplus}(\alpha, \beta ; \eta, \tau, v)=\mathcal{J}(y(u), u),
$$

since the infimum is attained for the optimal control $u$, its corresponding state $y(u)$ and its exact flux $\nu \nabla y(u)$, and for $\alpha$ going to zero. Hence, (13) states that the exact lower bound of the majorant (11) coincides with the optimal value of the cost functional of the optimal control problem. Therefore, we have the estimate

$$
\mathcal{J}(y(u), u) \leq \mathcal{J}^{\oplus}(\alpha, \beta ; \eta, \tau, v) \quad \forall \eta \in V, \boldsymbol{\tau} \in H(\operatorname{div}, \Omega), v \in U_{\mathrm{ad}}, \alpha, \beta>0,
$$

see [7, 35]. 


\section{Minorants for the Cost Functional}

In this work, we enrich the derivation of guaranteed upper bounds for the discretization error in the state and the control of problem (11)-(3) by obtaining fully computable lower bounds (minorants) for the cost functional $\mathcal{J}$. For any $\eta \in V$, we have that

$$
\mathcal{J}(y(v), v)=\frac{1}{2}\|y-\eta\|^{2}+\int_{\Omega}(y-\eta)\left(\eta-y_{d}\right) d \boldsymbol{x}+\frac{1}{2}\left\|\eta-y_{d}\right\|^{2}+\frac{\lambda}{2}\left\|v-u_{d}\right\|^{2}
$$

for all $v \in U_{\text {ad }}$. Since $\frac{1}{2}\|y-\eta\|^{2} \geq 0$, we can estimate $\mathcal{J}$ from below by

$$
\mathcal{J}(y(v), v) \geq \frac{1}{2}\left\|\eta-y_{d}\right\|^{2}+\frac{\lambda}{2}\left\|v-u_{d}\right\|^{2}+\int_{\Omega}(y-\eta)\left(\eta-y_{d}\right) d \boldsymbol{x} .
$$

Let $p_{\eta} \in V$ be the adjoint state corresponding to $\eta \in V$. Hence, $p_{\eta}$ solves the equation

$$
\int_{\Omega} \nu \nabla p_{\eta} \cdot \nabla w d \boldsymbol{x}=\int_{\Omega}\left(\eta-y_{d}\right) w d \boldsymbol{x} \quad \forall w \in V .
$$

By using (16), it follows for (15) that

$$
\begin{aligned}
\mathcal{J}(y(v), v) & \geq \frac{1}{2}\left\|\eta-y_{d}\right\|^{2}+\frac{\lambda}{2}\left\|v-u_{d}\right\|^{2}+\int_{\Omega}(\nabla y-\nabla \eta) \cdot \nu \nabla p_{\eta} d \boldsymbol{x} \\
& =\frac{1}{2}\left\|\eta-y_{d}\right\|^{2}+\frac{\lambda}{2}\left\|v-u_{d}\right\|^{2}+\int_{\Omega}(\nu \nabla y-\nu \nabla \eta) \cdot \nabla p_{\eta} d \boldsymbol{x} .
\end{aligned}
$$

Since $y=y(v)$ solves the variational formulation

$$
\int_{\Omega} \nu \nabla y \cdot \nabla w d \boldsymbol{x}=\int_{\Omega}(f+v) w d \boldsymbol{x} \quad \forall w \in V
$$

of the boundary value problem (2), we obtain

$$
\mathcal{J}(y(v), v) \geq \frac{1}{2}\left\|\eta-y_{d}\right\|^{2}+\frac{\lambda}{2}\left\|v-u_{d}\right\|^{2}+\int_{\Omega}(f+v) p_{\eta} d \boldsymbol{x}-\int_{\Omega} \nu \nabla \eta \cdot \nabla p_{\eta} d \boldsymbol{x} .
$$

For any $\tau \in H(\operatorname{div}, \Omega)$, the identity

$$
\int_{\Omega} \operatorname{div} \boldsymbol{\tau} w d \boldsymbol{x}=-\int_{\Omega} \boldsymbol{\tau} \cdot \nabla w d \boldsymbol{x} \quad \forall w \in V
$$

is valid, which yields

$$
\begin{aligned}
\mathcal{J}(y(v), v) \geq & \frac{1}{2}\left\|\eta-y_{d}\right\|^{2}+\frac{\lambda}{2}\left\|v-u_{d}\right\|^{2}+\int_{\Omega}(f+v) p_{\eta} d \boldsymbol{x}-\int_{\Omega} \nu \nabla \eta \cdot \nabla p_{\eta} d \boldsymbol{x} \\
& +\int_{\Omega} \operatorname{div} \boldsymbol{\tau} p_{\eta} d \boldsymbol{x}+\int_{\Omega} \boldsymbol{\tau} \cdot \nabla p_{\eta} d \boldsymbol{x} \\
= & \frac{1}{2}\left\|\eta-y_{d}\right\|^{2}+\frac{\lambda}{2}\left\|v-u_{d}\right\|^{2}+\int_{\Omega}(f+v+\operatorname{div} \boldsymbol{\tau}) p_{\eta} d \boldsymbol{x}+\int_{\Omega}(\boldsymbol{\tau}-\nu \nabla \eta) \cdot \nabla p_{\eta} d \boldsymbol{x}
\end{aligned}
$$

for all $v \in U_{\text {ad }}$. Moreover,

$$
\begin{aligned}
\mathcal{J}(y(u), u)=\inf _{v \in U_{\mathrm{ad}}} \mathcal{J}(y(v), v) \geq & \frac{1}{2}\left\|\eta-y_{d}\right\|^{2}+\int_{\Omega}(f+\operatorname{div} \boldsymbol{\tau}) p_{\eta} d \boldsymbol{x}+\int_{\Omega}(\boldsymbol{\tau}-\nu \nabla \eta) \cdot \nabla p_{\eta} d \boldsymbol{x} \\
& +\inf _{v \in U_{\mathrm{ad}}}\left(\int_{\Omega} v p_{\eta} d \boldsymbol{x}+\frac{\lambda}{2}\left\|v-u_{d}\right\|^{2}\right) .
\end{aligned}
$$

The control $v \in U_{\text {ad }}$ can be defined via the projection formula

$$
\mathbb{P}_{[a, b]}(u):=\min \{b, \max \{a, u\}\}
$$

for all $a, b \in \mathbb{R}$ with $a \leq b$ and $u \in \mathbb{R}$, which projects $\mathbb{R}$ on the interval $[a, b]$. It is given by

$$
v(\boldsymbol{x})=\mathbb{P}_{\left[u_{a}(\boldsymbol{x}), u_{b}(\boldsymbol{x})\right]}\left\{u_{d}(\boldsymbol{x})-\frac{1}{\lambda} p(\boldsymbol{x})\right\},
$$


where $p=p(v)$ is here the adjoint state corresponding to $v$ and $y(v)$. Let us denote by $v_{p_{\eta}} \in U_{\text {ad }}$ the control corresponding to the adjoint state $p_{\eta}$, i.e.,

$$
v_{p_{\eta}}(\boldsymbol{x})=\mathbb{P}_{\left[u_{a}(\boldsymbol{x}), u_{b}(\boldsymbol{x})\right]}\left\{u_{d}(\boldsymbol{x})-\frac{1}{\lambda} p_{\eta}(\boldsymbol{x})\right\}
$$

for almost every $\boldsymbol{x} \in \Omega$. By adding and subtracting $v_{p_{\eta}}$, we obtain

$$
\begin{aligned}
\mathcal{J}(y(v), v) \geq & \frac{1}{2}\left\|\eta-y_{d}\right\|^{2}+\frac{\lambda}{2}\left\|v_{p_{\eta}}-u_{d}\right\|^{2}+\frac{\lambda}{2}\left\|v-v_{p_{\eta}}\right\|^{2}+\lambda \int_{\Omega}\left(v-v_{p_{\eta}}\right)\left(v_{p_{\eta}}-u_{d}\right) d \boldsymbol{x} \\
& +\int_{\Omega}\left(v-v_{p_{\eta}}\right) p_{\eta} d \boldsymbol{x}+\int_{\Omega}\left(f+v_{p_{\eta}}+\operatorname{div} \boldsymbol{\tau}\right) p_{\eta} d \boldsymbol{x}+\int_{\Omega}(\boldsymbol{\tau}-\nu \nabla \eta) \cdot \nabla p_{\eta} d \boldsymbol{x}
\end{aligned}
$$

for all $v \in U_{\text {ad }}$. Since $\frac{\lambda}{2}\left\|v-v_{p_{\eta}}\right\|^{2} \geq 0$, we get that

$$
\begin{aligned}
\mathcal{J}(y(v), v) \geq & \frac{1}{2}\left\|\eta-y_{d}\right\|^{2}+\frac{\lambda}{2}\left\|v_{p_{\eta}}-u_{d}\right\|^{2}+\int_{\Omega}\left(v-v_{p_{\eta}}\right)\left(p_{\eta}+\lambda\left(v_{p_{\eta}}-u_{d}\right)\right) d \boldsymbol{x} \\
& +\int_{\Omega}\left(f+v_{p_{\eta}}+\operatorname{div} \boldsymbol{\tau}\right) p_{\eta} d \boldsymbol{x}+\int_{\Omega}(\boldsymbol{\tau}-\nu \nabla \eta) \cdot \nabla p_{\eta} d \boldsymbol{x} .
\end{aligned}
$$

Due to the variational inequality

$$
\int_{\Omega}\left(v-v_{p_{\eta}}\right)\left(p_{\eta}+\lambda\left(v_{p_{\eta}}-u_{d}\right)\right) d \boldsymbol{x} \geq 0 \quad \forall v \in U_{\mathrm{ad}}
$$

we obtain

$$
\mathcal{J}(y(v), v) \geq \frac{1}{2}\left\|\eta-y_{d}\right\|^{2}+\frac{\lambda}{2}\left\|v_{p_{\eta}}-u_{d}\right\|^{2}+\int_{\Omega}\left(f+v_{p_{\eta}}+\operatorname{div} \boldsymbol{\tau}\right) p_{\eta} d \boldsymbol{x}+\int_{\Omega}(\boldsymbol{\tau}-\nu \nabla \eta) \cdot \nabla p_{\eta} d \boldsymbol{x} .
$$

Now, let us introduce an arbitrary $\zeta \in V$ and its corresponding control $v_{\zeta} \in U_{\text {ad }}$, which can be computed by the projection formula as follows:

$$
v_{\zeta}(\boldsymbol{x})=\mathbb{P}_{\left[u_{a}(\boldsymbol{x}), u_{b}(\boldsymbol{x})\right]}\left\{u_{d}(\boldsymbol{x})-\frac{1}{\lambda} \zeta(\boldsymbol{x})\right\} .
$$

Hence, the following variational inequality is satisfied:

$$
\int_{\Omega}\left(v-v_{\zeta}\right)\left(\zeta+\lambda\left(v_{\zeta}-u_{d}\right)\right) d \boldsymbol{x} \geq 0 \quad \forall v \in U_{\mathrm{ad}} .
$$

Adding and subtracting $v_{\zeta} \in U_{\text {ad }}$ leads to

$$
\begin{aligned}
\mathcal{J}(y(v), v) \geq & \frac{1}{2}\left\|\eta-y_{d}\right\|^{2}+\frac{\lambda}{2}\left\|v_{\zeta}-u_{d}\right\|^{2}+\lambda \int_{\Omega}\left(v_{p_{\eta}}-v_{\zeta}\right)\left(v_{\zeta}-u_{d}\right) d \boldsymbol{x} \\
& +\int_{\Omega}\left(f+v_{\zeta}+\operatorname{div} \boldsymbol{\tau}\right) p_{\eta} d \boldsymbol{x}+\int_{\Omega}(\boldsymbol{\tau}-\nu \nabla \eta) \cdot \nabla p_{\eta} d \boldsymbol{x}+\int_{\Omega}\left(v_{p_{\eta}}-v_{\zeta}\right) p_{\eta} d \boldsymbol{x} \\
\geq & \frac{1}{2}\left\|\eta-y_{d}\right\|^{2}+\frac{\lambda}{2}\left\|v_{\zeta}-u_{d}\right\|^{2}+\int_{\Omega}\left(v_{p_{\eta}}-v_{\zeta}\right)\left(p_{\eta}-\zeta\right) d \boldsymbol{x} \\
& +\int_{\Omega}\left(f+v_{\zeta}+\operatorname{div} \boldsymbol{\tau}\right) p_{\eta} d \boldsymbol{x}+\int_{\Omega}(\boldsymbol{\tau}-\nu \nabla \eta) \cdot \nabla p_{\eta} d \boldsymbol{x},
\end{aligned}
$$

since $\frac{\lambda}{2}\left\|v_{p_{\eta}}-v_{\zeta}\right\|^{2} \geq 0$ and the variational inequality (22) is valid. Next, we add and subtract the arbitrary $\zeta \in V$ leading to the following estimate:

$$
\begin{aligned}
\mathcal{J}(y(v), v) \geq & \frac{1}{2}\left\|\eta-y_{d}\right\|^{2}+\frac{\lambda}{2}\left\|v_{\zeta}-u_{d}\right\|^{2}+\int_{\Omega}\left(v_{p_{\eta}}-v_{\zeta}\right)\left(p_{\eta}-\zeta\right) d \boldsymbol{x} \\
& +\int_{\Omega}\left(f+v_{\zeta}+\operatorname{div} \boldsymbol{\tau}\right) \zeta d \boldsymbol{x}+\int_{\Omega}(\boldsymbol{\tau}-\nu \nabla \eta) \cdot \nabla \zeta d \boldsymbol{x} \\
& +\int_{\Omega}\left(f+v_{\zeta}+\operatorname{div} \boldsymbol{\tau}\right)\left(p_{\eta}-\zeta\right) d \boldsymbol{x}+\int_{\Omega}(\boldsymbol{\tau}-\nu \nabla \eta) \cdot \nabla\left(p_{\eta}-\zeta\right) d \boldsymbol{x} .
\end{aligned}
$$

The following result provides an estimate for the error in the control: 
Lemma 1. Let $v_{p_{\eta}} \in U_{\mathrm{ad}}$ and $v_{\zeta} \in U_{\mathrm{ad}}$ satisfy the variational inequalities (20) and (22), respectively. Then, the error between $v_{p_{\eta}}$ and $v_{\zeta}$ can be estimated by

$$
\left\|v_{p_{\eta}}-v_{\zeta}\right\| \leq \frac{1}{\lambda}\left\|p_{\eta}-\zeta\right\| .
$$

Proof. Adding the variational inequalities

$$
\int_{\Omega}\left(v_{p_{\eta}}-v_{\zeta}\right)\left(\zeta+\lambda\left(v_{\zeta}-u_{d}\right)\right) d \boldsymbol{x} \geq 0 \quad \text { and } \quad \int_{\Omega}\left(v_{\zeta}-v_{p_{\eta}}\right)\left(p_{\eta}+\lambda\left(v_{p_{\eta}}-u_{d}\right)\right) d \boldsymbol{x} \geq 0
$$

yields the inequality

$$
\int_{\Omega}\left(v_{p_{\eta}}-v_{\zeta}\right)\left(\zeta-p_{\eta}\right) d \boldsymbol{x} \geq \lambda \int_{\Omega}\left(v_{p_{\eta}}-v_{\zeta}\right)^{2} d \boldsymbol{x} .
$$

By applying the Cauchy-Schwarz inequality, we obtain

$$
\left\|v_{p_{\eta}}-v_{\zeta}\right\|\left\|p_{\eta}-\zeta\right\| \geq \int_{\Omega}\left(v_{p_{\eta}}-v_{\zeta}\right)\left(\zeta-p_{\eta}\right) d \boldsymbol{x} \geq \lambda\left\|v_{p_{\eta}}-v_{\zeta}\right\|^{2},
$$

which finally leads to the estimate (24).

In order to formulate, a computable lower bound for the cost functional, we need to prove a computable upper bound for the error in the adjoint state, which is presented in the following theorem:

Theorem 1. Let $y_{d} \in L^{2}(\Omega)$ be given and let $p_{\eta} \in V$ meet equation (16) with $\eta \in V$ and $\nu(\cdot)$ satisfying assumption (4). For any $\zeta \in V$, we have that

$$
\left\|\nabla\left(p_{\eta}-\zeta\right)\right\| \leq \frac{1}{\underline{\nu}}\left(C_{F}\left\|\eta-y_{d}+\operatorname{div} \boldsymbol{\rho}\right\|+\|\rho-\nu \nabla \zeta\|\right),
$$

where $\boldsymbol{\rho} \in H(\operatorname{div}, \Omega)$ and $C_{F}>0$ is the constant coming from the Friedrichs inequality.

Proof. Since the bilinear form of problem (16) is elliptic with ellipticity constant $\underline{\nu}$ and applying the Cauchy-Schwarz and Friedrichs inequalities, we get that

$$
\begin{aligned}
& \underline{\nu}\left\|\nabla\left(p_{\eta}-\zeta\right)\right\| \leq \sup _{0 \neq w \in V} \frac{\int_{\Omega} \nu \nabla\left(p_{\eta}-\zeta\right) \cdot \nabla w d \boldsymbol{x}}{\|\nabla w\|}=\sup _{0 \neq w \in V} \frac{\int_{\Omega}\left(\eta-y_{d}\right) w-\nu \nabla \zeta \cdot \nabla w d \boldsymbol{x}}{\|\nabla w\|} \\
& =\sup _{0 \neq w \in V} \frac{\int_{\Omega}\left(\eta-y_{d}+\operatorname{div} \boldsymbol{\rho}\right) w+(\boldsymbol{\rho}-\nu \nabla \zeta) \cdot \nabla w d \boldsymbol{x}}{\|\nabla w\|} \\
& \leq \sup _{0 \neq w \in V} \frac{\left\|\eta-y_{d}+\operatorname{div} \boldsymbol{\rho}\right\|\|w\|+\|\boldsymbol{\rho}-\nu \nabla \zeta\|\|\nabla w\|}{\|\nabla w\|} \\
& \leq C_{F}\left\|\eta-y_{d}+\operatorname{div} \boldsymbol{\rho}\right\|+\|\boldsymbol{\rho}-\nu \nabla \zeta\|
\end{aligned}
$$

where $\boldsymbol{\rho} \in H(\operatorname{div}, \Omega)$ satisfies identity (18). Hence, it follows the estimate (25).

By using the Cauchy-Schwarz and Friedrichs inequalities as well as Lemma 1, we can further estimate the inequality (23) from below as follows:

$$
\begin{aligned}
\mathcal{J}(y(v), v) \geq & \frac{1}{2}\left\|\eta-y_{d}\right\|^{2}+\frac{\lambda}{2}\left\|v_{\zeta}-u_{d}\right\|^{2}+\int_{\Omega}\left(f+v_{\zeta}+\operatorname{div} \boldsymbol{\tau}\right) \zeta d \boldsymbol{x}+\int_{\Omega}(\boldsymbol{\tau}-\nu \nabla \eta) \cdot \nabla \zeta d \boldsymbol{x} \\
& -\left\|v_{p_{\eta}}-v_{\zeta}\right\|\left\|p_{\eta}-\zeta\right\|-\left\|f+v_{\zeta}+\operatorname{div} \boldsymbol{\tau}\right\|\left\|p_{\eta}-\zeta\right\|-\|\boldsymbol{\tau}-\nu \nabla \eta\|\left\|\nabla\left(p_{\eta}-\zeta\right)\right\| \\
\geq & \frac{1}{2}\left\|\eta-y_{d}\right\|^{2}+\frac{\lambda}{2}\left\|v_{\zeta}-u_{d}\right\|^{2}+\int_{\Omega}\left(f+v_{\zeta}+\operatorname{div} \boldsymbol{\tau}\right) \zeta d \boldsymbol{x}+\int_{\Omega}(\boldsymbol{\tau}-\nu \nabla \eta) \cdot \nabla \zeta d \boldsymbol{x} \\
& -\frac{1}{\lambda}\left\|p_{\eta}-\zeta\right\|^{2}-\left(C_{F}\left\|f+v_{\zeta}+\operatorname{div} \boldsymbol{\tau}\right\|+\|\boldsymbol{\tau}-\nu \nabla \eta\|\right)\left\|\nabla\left(p_{\eta}-\zeta\right)\right\| \\
\geq & \frac{1}{2}\left\|\eta-y_{d}\right\|^{2}+\frac{\lambda}{2}\left\|v_{\zeta}-u_{d}\right\|^{2}+\int_{\Omega}\left(f+v_{\zeta}+\operatorname{div} \boldsymbol{\tau}\right) \zeta d \boldsymbol{x}+\int_{\Omega}(\boldsymbol{\tau}-\nu \nabla \eta) \cdot \nabla \zeta d \boldsymbol{x} \\
& -\frac{C_{F}^{2}}{\lambda}\left\|\nabla\left(p_{\eta}-\zeta\right)\right\|^{2}-\left(C_{F}\left\|f+v_{\zeta}+\operatorname{div} \boldsymbol{\tau}\right\|+\|\boldsymbol{\tau}-\nu \nabla \eta\|\right)\left\|\nabla\left(p_{\eta}-\zeta\right)\right\| .
\end{aligned}
$$


Now, applying Theorem 1 yields the following estimate:

$$
\mathcal{J}(y(v), v) \geq \mathcal{J}^{\ominus}\left(\eta, \zeta, \boldsymbol{\tau}, \boldsymbol{\rho}, v_{\zeta}\right) \quad \forall \eta, \zeta \in V, \boldsymbol{\tau}, \boldsymbol{\rho} \in H(\operatorname{div}, \Omega), v_{\zeta}=\mathbb{P}_{\left[u_{a}, u_{b}\right]}\left\{u_{d}-\frac{1}{\lambda} \zeta\right\}
$$

with the minorant

$$
\begin{aligned}
\mathcal{J}^{\ominus}(\eta, \zeta, & \left.\boldsymbol{\tau}, \boldsymbol{\rho}, v_{\zeta}\right)=\frac{1}{2}\left\|\eta-y_{d}\right\|^{2}+\frac{\lambda}{2}\left\|v_{\zeta}-u_{d}\right\|^{2}+\int_{\Omega}\left(f+v_{\zeta}+\operatorname{div} \boldsymbol{\tau}\right) \zeta d \boldsymbol{x} \\
& +\int_{\Omega}(\boldsymbol{\tau}-\nu \nabla \eta) \cdot \nabla \zeta d \boldsymbol{x}-\frac{1}{\underline{\nu}}\left(C_{F}\left\|\eta-y_{d}+\operatorname{div} \boldsymbol{\rho}\right\|+\|\boldsymbol{\rho}-\nu \nabla \zeta\|\right) \\
& \times\left(\frac{C_{F}^{3}}{\lambda \underline{\nu}}\left\|\eta-y_{d}+\operatorname{div} \boldsymbol{\rho}\right\|+\frac{C_{F}^{2}}{\lambda \underline{\nu}}\|\boldsymbol{\rho}-\nu \nabla \zeta\|+C_{F}\left\|f+v_{\zeta}+\operatorname{div} \boldsymbol{\tau}\right\|+\|\boldsymbol{\tau}-\nu \nabla \eta\|\right),
\end{aligned}
$$

where $\boldsymbol{\tau}, \boldsymbol{\rho} \in H(\operatorname{div}, \Omega)$. Note that the minorant is fully computable.

Theorem 2. The exact upper bound of the minorant $\mathcal{J}^{\ominus}$ defined in (27) coincides with the optimal value of the cost functional of problem (1)-(3), or, equivalently, of the optimality system (6)-(8), i.e.,

$$
\sup _{\substack{\eta, \zeta \in V, \boldsymbol{\tau}, \boldsymbol{\rho} \in H(\operatorname{div}, \Omega), v_{\zeta}=\mathbb{P}_{\left[u_{a}, u_{b}\right]}\left\{u_{d}-\frac{1}{\lambda} \zeta\right\}}} \mathcal{J}^{\ominus}\left(\eta, \zeta, \boldsymbol{\tau}, \boldsymbol{\rho}, v_{\zeta}\right)=\mathcal{J}(y(u), u) .
$$

Proof. The estimate (26) is valid for all $v \in U_{\text {ad }}$. Hence, also for the exact solution $u$, i.e.,

$$
\mathcal{J}(y(u), u)=\inf _{v \in U_{\text {ad }}} \mathcal{J}(y(v), v) \geq \mathcal{J}^{\ominus}\left(\eta, \zeta, \boldsymbol{\tau}, \boldsymbol{\rho}, v_{\zeta}\right)
$$

for all $\eta, \zeta \in V, \boldsymbol{\tau}, \boldsymbol{\rho} \in H(\operatorname{div}, \Omega)$ and for the control $v_{\zeta} \in U_{\mathrm{ad}}$, which depends on $\zeta$ and can be computed by the projection formula (21). For the exact solution $v_{\zeta}=u, \eta=y(u), \zeta=p(u)$, $\boldsymbol{\tau}=\nu \nabla y(u)$ and $\boldsymbol{\rho}=\nu \nabla p(u)$, the estimate is sharp, i.e.,

$$
\mathcal{J}^{\ominus}(y(u), p(u), \nu \nabla y(u), \nu \nabla p(u), u)=\frac{1}{2}\left\|y-y_{d}\right\|^{2}+\frac{\lambda}{2}\left\|u-u_{d}\right\|^{2}=\mathcal{J}(y(u), u) .
$$

\section{A Posteriori Error Estimates for Control and State}

In this section, we will derive guaranteed upper bounds for the discretization errors of the control and the state measured in the following norm:

$$
\|\| u-v\|\|^{2}:=\frac{1}{2}\|y(u)-y(v)\|^{2}+\frac{\lambda}{2}\|u-v\|^{2}
$$

making use of the ideas based on the work by Mikhlin [26] but generalized for the class of optimal control problems, see also 35 .

Theorem 3. For any control function $v \in U_{a d}$, we have the estimate

$$
\|u-v\| \|^{2} \leq \mathcal{J}(y(v), v)-\mathcal{J}(y(u), u) .
$$

Proof. We compute the difference

$$
\begin{aligned}
\mathcal{J}(y(v), v)-\mathcal{J}(y(u), u)=\frac{1}{2}\left\|y(v)-y_{d}\right\|^{2}-\frac{1}{2}\left\|y(u)-y_{d}\right\|^{2}+\frac{\lambda}{2}\left\|v-u_{d}\right\|^{2}-\frac{\lambda}{2}\left\|u-u_{d}\right\|^{2} \\
=\frac{1}{2} \int_{\Omega}\left(y(v)+y(u)-2 y_{d}\right)(y(v)-y(u)) d \boldsymbol{x}+\frac{\lambda}{2} \int_{\Omega}\left(v+u-2 u_{d}\right)(v-u) d \boldsymbol{x} \\
=\frac{1}{2} \int_{\Omega}\left(y(v)-y(u)+2 y(u)-2 y_{d}\right)(y(v)-y(u)) d \boldsymbol{x}+\frac{\lambda}{2} \int_{\Omega}\left(v-u+2 u-2 u_{d}\right)(v-u) d \boldsymbol{x} \\
=\frac{1}{2}\|y(u)-y(v)\|^{2}+\int_{\Omega}\left(y(u)-y_{d}\right)(y(v)-y(u)) d \boldsymbol{x}+\frac{\lambda}{2}\|u-v\|^{2}+\lambda \int_{\Omega}\left(u-u_{d}\right)(v-u) d \boldsymbol{x} .
\end{aligned}
$$


Since the exact adjoint state $p(u) \in V$ fulfills (7), we obtain the equation

$$
\begin{aligned}
& \mathcal{J}(y(v), v)-\mathcal{J}(y(u), u) \\
& \quad=\frac{1}{2}\|y(u)-y(v)\|^{2}+\int_{\Omega} \nu \nabla p(u)(\nabla y(v)-\nabla y(u)) d \boldsymbol{x}+\frac{\lambda}{2}\|u-v\|^{2}+\lambda \int_{\Omega}\left(u-u_{d}\right)(v-u) d \boldsymbol{x} \\
& \quad=\frac{1}{2}\|y(u)-y(v)\|^{2}+\int_{\Omega} \nabla p(u)(\nu \nabla y(v)-\nu \nabla y(u)) d \boldsymbol{x}+\frac{\lambda}{2}\|u-v\|^{2}+\lambda \int_{\Omega}\left(u-u_{d}\right)(v-u) d \boldsymbol{x} .
\end{aligned}
$$

From equations (6) and (17) follows that

$$
\begin{aligned}
& \mathcal{J}(y(v), v)-\mathcal{J}(y(u), u) \\
& \quad=\frac{1}{2}\|y(u)-y(v)\|^{2}+\int_{\Omega} p(u)(f+v-f-u) d \boldsymbol{x}+\frac{\lambda}{2}\|u-v\|^{2}+\lambda \int_{\Omega}\left(u-u_{d}\right)(v-u) d \boldsymbol{x} \\
& \quad=\|u-v\|^{2}+\int_{\Omega}\left(p(u)+\lambda\left(u-u_{d}\right)\right)(v-u) d \boldsymbol{x} .
\end{aligned}
$$

Since the variational inequality (8) is satisfied, i.e.,

$$
\int_{\Omega}\left(p(u)+\lambda\left(u-u_{d}\right)\right)(v-u) d \boldsymbol{x} \geq 0 \quad \forall v \in U_{\mathrm{ad}},
$$

we finally obtain the estimate (31).

Theorem 4. For any $\zeta \in V$, let $v_{\zeta} \in U_{a d}$ be given by the projection formula (21). Then, we obtain the following error majorant:

$$
\left\|u-v_{\zeta} \mid\right\|^{2} \leq \mathcal{M}^{\oplus}\left(\alpha, \beta ; \eta, \zeta, \boldsymbol{\tau}, \boldsymbol{\rho}, v_{\zeta}\right):=\mathcal{J}^{\oplus}\left(\alpha, \beta ; \eta, \boldsymbol{\tau}, v_{\zeta}\right)-\mathcal{J}^{\ominus}\left(\eta, \zeta, \boldsymbol{\tau}, \boldsymbol{\rho}, v_{\zeta}\right)
$$

with

$$
\begin{aligned}
\mathcal{M}^{\oplus}(\alpha, \beta ; \eta, \zeta, & \left.\boldsymbol{\tau}, \boldsymbol{\rho}, v_{\zeta}\right)=\frac{\alpha}{2}\left\|\eta-y_{d}\right\|^{2}+\frac{(1+\alpha)(1+\beta) C_{F}^{2}}{2 \alpha \underline{\nu}^{2}}\|\boldsymbol{\tau}-\nu \nabla \eta\|^{2} \\
& +\frac{(1+\alpha)(1+\beta) C_{F}^{4}}{2 \alpha \beta \underline{\nu}^{2}}\left\|f+v_{\zeta}+\operatorname{div} \boldsymbol{\tau}\right\|^{2}-\int_{\Omega}\left(f+v_{\zeta}+\operatorname{div} \boldsymbol{\tau}\right) \zeta d \boldsymbol{x} \\
& -\int_{\Omega}(\boldsymbol{\tau}-\nu \nabla \eta) \cdot \nabla \zeta d \boldsymbol{x}+\frac{1}{\underline{\nu}}\left(C_{F}\left\|\eta-y_{d}+\operatorname{div} \boldsymbol{\rho}\right\|+\|\boldsymbol{\rho}-\nu \nabla \zeta\|\right) \\
& \times\left(\frac{C_{F}^{3}}{\lambda \underline{\nu}}\left\|\eta-y_{d}+\operatorname{div} \boldsymbol{\rho}\right\|+\frac{C_{F}^{2}}{\lambda \underline{\nu}}\|\boldsymbol{\rho}-\nu \nabla \zeta\|+C_{F}\left\|f+v_{\zeta}+\operatorname{div} \boldsymbol{\tau}\right\|+\|\boldsymbol{\tau}-\nu \nabla \eta\|\right)
\end{aligned}
$$

for arbitrary $\eta \in V, \boldsymbol{\tau}, \boldsymbol{\rho} \in H(\operatorname{div}, \Omega)$ and $\alpha, \beta>0$.

Proof. Using (31), we obtain the estimate

$$
\left\|u-v_{\zeta} \mid\right\|^{2} \leq \mathcal{J}\left(y\left(v_{\zeta}\right), v_{\zeta}\right)-\mathcal{J}(y(u), u) .
$$

Applying (10) and (29) finally leads to the estimate (32).

Proposition 1. The majorant $\mathcal{M}^{\oplus}\left(\alpha, \beta ; \eta, \zeta, \boldsymbol{\tau}, \boldsymbol{\rho}, v_{\zeta}\right)$ defined in (32) attains the exact lower bound on the exact solution of the optimal control problem (1)-(3), or, equivalently, of the optimality system (6)-(8), i.e.,

$$
\inf _{\substack{\eta, \zeta \in V, \boldsymbol{\tau}, \boldsymbol{\rho} \in H(\operatorname{div}, \Omega), v_{\zeta}=\mathbb{P}_{\left[u_{a}, u_{b}\right]}\left\{u_{d}-\frac{1}{\lambda} \zeta\right\}, \alpha, \beta>0}} \mathcal{M}^{\oplus}\left(\alpha, \beta ; \eta, \zeta, \boldsymbol{\tau}, \boldsymbol{\rho}, v_{\zeta}\right)=0 .
$$

The infimum is attained for $v_{\zeta}=u, \eta=y(u), \zeta=p(u), \boldsymbol{\tau}=\nu \nabla y(u)$ and $\boldsymbol{\rho}=\nu \nabla p(u)$.

Proof. We have that

$$
\mathcal{M}^{\oplus}(\alpha, \beta ; y(u), p(u), \nu \nabla y(u), \nu \nabla p(u), u)=\frac{\alpha}{2}\left\|y(u)-y_{d}\right\|^{2},
$$

which is zero if we let $\alpha$ go to zero. 
Although the majorant $\mathcal{M}^{\oplus}$ is a guaranteed, computable and sharp upper estimate for the discretization error in the combined norm, it only decreases with order $h$ when discretizing the mesh. However, the combined norm $\||\cdot|\|$ is an $L^{2}$-norm, and, hence, decreases with order $h^{2}$. So the majorant $\mathcal{M}^{\oplus}$ is an overestimation for the combined norm. Now, we introduce another norm which is a weighted $H^{1}$-norm for the state depending on the corresponding control. More precisely, we derive an estimate for the discretization error measured in the following norm:

$$
\|u-v\|\left\|_{1}^{2}:=\frac{1}{2}\right\| y(u)-y(v)\left\|^{2}+\frac{2 \lambda \underline{\nu}^{2}}{C_{F}^{2}}\right\| \nabla y(u)-\nabla y(v) \|^{2} .
$$

Theorem 5. For any control function $v \in U_{a d}$, we have the estimate

$$
\|u-v\| \|_{1}^{2} \leq \mathcal{J}(y(v), v)-\mathcal{J}(y(u), u)+\frac{3 \lambda}{2 C_{F}^{2}}\left(\|\boldsymbol{\tau}-\nu \nabla \eta\|+C_{F}\|f+v+\operatorname{div} \boldsymbol{\tau}\|\right)^{2} .
$$

Proof. Let $\delta>0$ be an arbitrary but fixed parameter. Adding and subtracting $\nabla \eta$ as well as applying triangle inequality for $\frac{\underline{\nu}^{2}}{C_{F}^{2} \delta}\|\nabla y(u)-\nabla y(v)\|^{2}$, we derive the following estimate:

$$
\frac{\underline{\nu}^{2}}{C_{F}^{2} \delta}\|\nabla y(u)-\nabla y(v)\|^{2} \leq \frac{\underline{\nu}^{2}}{2 C_{F}^{2} \delta}\left(\|\nabla y(u)-\nabla \eta\|^{2}+\|\nabla y(v)-\nabla \eta\|^{2}\right) .
$$

Using (12), adding and subtracting $v$ as well as applying twice triangle inequality, we arrive at the estimate

$$
\begin{aligned}
\frac{\underline{\nu}^{2}}{C_{F}^{2} \delta}\|\nabla y(u)-\nabla y(v)\|^{2} \leq \frac{1}{2 C_{F}^{2} \delta}( & \left(\|\boldsymbol{\tau}-\nu \nabla \eta\|+C_{F}\|f+v+\operatorname{div} \boldsymbol{\tau}\|+C_{F}\|u-v\|\right)^{2} \\
& \left.+\left(\|\boldsymbol{\tau}-\nu \nabla \eta\|+C_{F}\|f+v+\operatorname{div} \boldsymbol{\tau}\|\right)^{2}\right) \\
\leq & \frac{3}{4 C_{F}^{2} \delta}\left(\|\boldsymbol{\tau}-\nu \nabla \eta\|+C_{F}\|f+v+\operatorname{div} \boldsymbol{\tau}\|\right)^{2}+\frac{1}{4 \delta}\|u-v\|^{2} .
\end{aligned}
$$

By using (31) and the previous estimate, we derive the inequality

$$
\begin{aligned}
\|u-v\| \|^{2} & +\frac{\underline{\nu}^{2}}{C_{F}^{2} \delta}\|\nabla y(u)-\nabla y(v)\|^{2}-\frac{1}{4 \delta}\|u-v\|^{2} \\
& =\frac{1}{2}\|y(u)-y(v)\|^{2}+\frac{\underline{\nu}^{2}}{C_{F}^{2} \delta}\|\nabla y(u)-\nabla y(v)\|^{2}+\left(\frac{\lambda}{2}-\frac{1}{4 \delta}\right)\|u-v\|^{2} \\
& \leq \mathcal{J}(y(v), v)-\mathcal{J}(y(u), u)+\frac{3}{4 C_{F}^{2} \delta}\left(\|\boldsymbol{\tau}-\nu \nabla \eta\|+C_{F}\|f+v+\operatorname{div} \boldsymbol{\tau}\|\right)^{2} .
\end{aligned}
$$

Finally, choosing $\delta=1 /(2 \lambda)$ yields the estimate (34).

Theorem 6. For any $\zeta \in V$, let $v_{\zeta} \in U_{a d}$ be given by the projection formula (21). Then, we obtain the following error majorant:

$$
\left\|u-v_{\zeta}\right\|_{1}^{2} \leq \mathcal{M}_{1}^{\oplus}\left(\alpha, \beta ; \eta, \zeta, \boldsymbol{\tau}, \boldsymbol{\rho}, v_{\zeta}\right)
$$

for arbitrary $\eta \in V, \boldsymbol{\tau}, \boldsymbol{\rho} \in H(\operatorname{div}, \Omega)$ and $\alpha, \beta>0$, where

$$
\begin{aligned}
\mathcal{M}_{1}^{\oplus}(\alpha, \beta ; \eta, \zeta, & \left.\boldsymbol{\tau}, \boldsymbol{\rho}, v_{\zeta}\right)=\frac{\alpha}{2}\left\|\eta-y_{d}\right\|^{2}+\frac{(1+\alpha)(1+\beta) C_{F}^{2}}{2 \alpha \underline{\nu}^{2}}\|\boldsymbol{\tau}-\nu \nabla \eta\|^{2} \\
& +\frac{(1+\alpha)(1+\beta) C_{F}^{4}}{2 \alpha \beta \underline{\nu}^{2}}\left\|f+v_{\zeta}+\operatorname{div} \boldsymbol{\tau}\right\|^{2}-\int_{\Omega}\left(f+v_{\zeta}+\operatorname{div} \boldsymbol{\tau}\right) \zeta d \boldsymbol{x} \\
& -\int_{\Omega}(\boldsymbol{\tau}-\nu \nabla \eta) \cdot \nabla \zeta d \boldsymbol{x}+\frac{1}{\underline{\nu}}\left(C_{F}\left\|\eta-y_{d}+\operatorname{div} \boldsymbol{\rho}\right\|+\|\boldsymbol{\rho}-\nu \nabla \zeta\|\right) \\
& \times\left(\frac{C_{F}^{3}}{\lambda \underline{\nu}}\left\|\eta-y_{d}+\operatorname{div} \boldsymbol{\rho}\right\|+\frac{C_{F}^{2}}{\lambda \underline{\nu}}\|\boldsymbol{\rho}-\nu \nabla \zeta\|+C_{F}\left\|f+v_{\zeta}+\operatorname{div} \boldsymbol{\tau}\right\|+\|\boldsymbol{\tau}-\nu \nabla \eta\|\right) \\
& +\frac{3 \lambda}{2 C_{F}^{2}}\left(\|\boldsymbol{\tau}-\nu \nabla \eta\|+C_{F}\left\|f+v_{\zeta}+\operatorname{div} \boldsymbol{\tau}\right\|\right)^{2}
\end{aligned}
$$


Proof. Applying (34) as well as (10) and (29) finally leads to the estimate (35).

Proposition 2. The majorant $\mathcal{M}_{1}^{\oplus}\left(\alpha, \beta ; \eta, \zeta, \boldsymbol{\tau}, \boldsymbol{\rho}, v_{\zeta}\right)$ defined in (35) attains the exact lower bound on the exact solution of the optimal control problem (1)-(3), or, equivalently, of the optimality system (6)-(8), i.e.,

$$
\inf _{\substack{\eta, \zeta \in V, \boldsymbol{\tau}, \boldsymbol{\rho} \in H(\operatorname{div}, \Omega), v_{\zeta}=\mathbb{P}_{\left[u_{a}, u_{b}\right]}\left\{u_{d}-\frac{1}{\lambda} \zeta\right\}, \alpha, \beta>0}} \mathcal{M}_{1}^{\oplus}\left(\alpha, \beta ; \eta, \zeta, \boldsymbol{\tau}, \boldsymbol{\rho}, v_{\zeta}\right)=0 .
$$

The infimum is attained for $v_{\zeta}=u, \eta=y(u), \zeta=p(u), \boldsymbol{\tau}=\nu \nabla y(u)$ and $\boldsymbol{\rho}=\nu \nabla p(u)$.

Proof. We have that

$$
\mathcal{M}_{1}^{\oplus}(\alpha, \beta ; y(u), p(u), \nu \nabla y(u), \nu \nabla p(u), u)=\frac{\alpha}{2}\left\|y(u)-y_{d}\right\|^{2},
$$

which is zero if we let $\alpha$ go to zero.

\section{The Unconstrained Case}

In the unconstrained case, we have that $U_{\mathrm{ad}}=L^{2}(\Omega)$, i.e., $v_{\zeta}$ satisfies the optimality condition

$$
\zeta+\lambda\left(v_{\zeta}-u_{d}\right)=0 \quad \text { in } \Omega,
$$

or, equivalently, $v_{\zeta}=u_{d}-\frac{1}{\lambda} \zeta$ in $\Omega$. The majorant (11) and minorant (27) simplify to

$$
\begin{aligned}
\mathcal{J}^{\oplus}(\alpha, \beta ; \eta, \zeta, \boldsymbol{\tau}):= & \frac{1+\alpha}{2}\left\|\eta-y_{d}\right\|^{2}+\frac{(1+\alpha)(1+\beta) C_{F}^{2}}{2 \alpha \underline{\nu}^{2}}\|\boldsymbol{\tau}-\nu \nabla \eta\|^{2} \\
& +\frac{(1+\alpha)(1+\beta) C_{F}^{4}}{2 \alpha \beta \underline{\nu}^{2}}\left\|f+u_{d}-\frac{1}{\lambda} \zeta+\operatorname{div} \boldsymbol{\tau}\right\|^{2}+\frac{1}{2 \lambda}\|\zeta\|^{2},
\end{aligned}
$$

where $\mathcal{J}^{\oplus}(\alpha, \beta ; \eta, \zeta, \boldsymbol{\tau})=\mathcal{J}^{\oplus}\left(\alpha, \beta ; \eta, \boldsymbol{\tau}, v_{\zeta}\right)$, and

$$
\begin{aligned}
& \mathcal{J}^{\ominus}(\eta, \zeta, \boldsymbol{\tau}, \boldsymbol{\rho})=\frac{1}{2}\left\|\eta-y_{d}\right\|^{2}+\frac{1}{2 \lambda}\|\zeta\|^{2}+\int_{\Omega}\left(f+u_{d}-\frac{1}{\lambda} \zeta+\operatorname{div} \boldsymbol{\tau}\right) \zeta d \boldsymbol{x} \\
& \quad+\int_{\Omega}(\boldsymbol{\tau}-\nu \nabla \eta) \cdot \nabla \zeta d \boldsymbol{x}-\frac{1}{\underline{\nu}}\left(C_{F}\left\|\eta-y_{d}+\operatorname{div} \boldsymbol{\rho}\right\|+\|\boldsymbol{\rho}-\nu \nabla \zeta\|\right) \\
& \quad \times\left(\frac{C_{F}^{3}}{\lambda \underline{\underline{\nu}}}\left\|\eta-y_{d}+\operatorname{div} \boldsymbol{\rho}\right\|+\frac{C_{F}^{2}}{\lambda \underline{\nu}}\|\boldsymbol{\rho}-\nu \nabla \zeta\|+C_{F}\left\|f+u_{d}-\frac{1}{\lambda} \zeta+\operatorname{div} \boldsymbol{\tau}\right\|+\|\boldsymbol{\tau}-\nu \nabla \eta\|\right),
\end{aligned}
$$

respectively. We obtain the following estimate using minorant (38):

$$
\mathcal{J}(y(u), u) \geq \mathcal{J}^{\ominus}(\eta, \zeta, \boldsymbol{\tau}, \boldsymbol{\rho}) \quad \forall \eta, \zeta \in V \forall \boldsymbol{\tau}, \boldsymbol{\rho} \in H(\operatorname{div}, \Omega) .
$$

The minorant $\mathcal{J}^{\ominus}$ is sharp, i.e.,

$$
\sup _{\eta, \zeta \in V, \boldsymbol{\tau}, \boldsymbol{\rho} \in H(\operatorname{div}, \Omega)} \mathcal{J}^{\ominus}(\eta, \zeta, \boldsymbol{\tau}, \boldsymbol{\rho})=\mathcal{J}(y(u), u),
$$

since the supremum of $\mathcal{J}^{\ominus}$ is attained for the optimal state $\eta=y$ and adjoint state $\zeta=p$, and their corresponding exact fluxes $\boldsymbol{\tau}=\nu \nabla y$ and $\boldsymbol{\rho}=\nu \nabla p$. The optimal control is given by $u=u_{d}-\frac{1}{\lambda} p$. In the unconstrained case, the proof of Theorem 3 provides not only an inequality but even an equation. This result is presented in the following theorem:

Theorem 7. For any control function $v \in L^{2}(\Omega)$, we have that

$$
\|u-v\| \|^{2}=\mathcal{J}(y(v), v)-\mathcal{J}(y(u), u) .
$$

Proof. The equation (41) follows by repeating the proof of Theorem 3 with applying equation (9) instead of inequality (8). 
The norm ||$|\cdot|||$ defined in (30) can be represented in terms of the state and the adjoint state (instead of the control) by using the optimality condition (9) for $u$ and $v$ as follows:

$$
\begin{aligned}
\|u-v\| \|^{2} & =\frac{1}{2}\|y(u)-y(v)\|^{2}+\frac{\lambda}{2}\|u-v\|^{2} \\
& =\frac{1}{2}\|y(u)-y(v)\|^{2}+\frac{\lambda}{2}\left\|u_{d}-\frac{1}{\lambda} p(u)-\left(u_{d}-\frac{1}{\lambda} p(v)\right)\right\|^{2} \\
& =\frac{1}{2}\|y(u)-y(v)\|^{2}+\frac{1}{2 \lambda}\|p(u)-p(v)\|^{2} .
\end{aligned}
$$

Next, we present similar results to those of Theorem 4 and Proposition 1 for the unconstrained case.

Theorem 8. Let $U_{a d}=L^{2}(\Omega)$. For any $\eta, \zeta \in V$, we obtain the following error majorant:

$$
\left\|u-v_{\zeta}\right\|^{2} \leq \mathcal{M}^{\oplus}(\alpha, \beta ; \eta, \zeta, \boldsymbol{\tau}, \boldsymbol{\rho}):=\mathcal{J}^{\oplus}(\alpha, \beta ; \eta, \zeta, \boldsymbol{\tau})-\mathcal{J}^{\ominus}(\eta, \zeta, \boldsymbol{\tau}, \boldsymbol{\rho})
$$

$$
\text { with }\left.\left\|u-v_{\zeta}\right\|\right|^{2}=\|\| u-u_{d}+\frac{1}{\lambda} \zeta\|\|^{2} \text { and }
$$

$$
\begin{aligned}
\mathcal{M}^{\oplus}(\alpha, \beta ; & \eta, \zeta, \boldsymbol{\tau}, \boldsymbol{\rho})=\frac{\alpha}{2}\left\|\eta-y_{d}\right\|^{2}+\frac{(1+\alpha)(1+\beta) C_{F}^{2}}{2 \alpha \underline{\nu}^{2}}\|\boldsymbol{\tau}-\nu \nabla \eta\|^{2} \\
& +\frac{(1+\alpha)(1+\beta) C_{F}^{4}}{2 \alpha \beta \underline{\nu}^{2}}\left\|f+u_{d}-\frac{1}{\lambda} \zeta+\operatorname{div} \boldsymbol{\tau}\right\|^{2}-\int_{\Omega}\left(f+u_{d}-\frac{1}{\lambda} \zeta+\operatorname{div} \boldsymbol{\tau}\right) \zeta d \boldsymbol{x} \\
& -\int_{\Omega}(\boldsymbol{\tau}-\nu \nabla \eta) \cdot \nabla \zeta d \boldsymbol{x}+\frac{1}{\underline{\nu}}\left(C_{F}\left\|\eta-y_{d}+\operatorname{div} \boldsymbol{\rho}\right\|+\|\boldsymbol{\rho}-\nu \nabla \zeta\|\right) \\
& \times\left(\frac{C_{F}^{3}}{\lambda \underline{\nu}}\left\|\eta-y_{d}+\operatorname{div} \boldsymbol{\rho}\right\|+\frac{C_{F}^{2}}{\lambda \underline{\nu}}\|\boldsymbol{\rho}-\nu \nabla \zeta\|+C_{F}\left\|f+u_{d}-\frac{1}{\lambda} \zeta+\operatorname{div} \boldsymbol{\tau}\right\|+\|\boldsymbol{\tau}-\nu \nabla \eta\|\right)
\end{aligned}
$$

where $\boldsymbol{\tau}, \boldsymbol{\rho} \in H(\operatorname{div}, \Omega)$ are arbitrary and $\alpha, \beta>0$.

Proof. The estimate follows by applying (41) and then using the estimates (10) and (39).

Proposition 3. Let $U_{a d}=L^{2}(\Omega)$. The majorant $\mathcal{M}^{\oplus}(\alpha, \beta ; \eta, \zeta, \boldsymbol{\tau}, \boldsymbol{\rho})$ defined in 43 attains the exact lower bound on the exact solution of the optimal control problem (1)-(2), or, equivalently, of the optimality system (6), (7) and (9), i.e.,

$$
\inf _{\substack{\eta, \zeta \in V, \boldsymbol{\tau}, \boldsymbol{\rho} \in H(\operatorname{div}, \Omega), \alpha, \beta>0}} \mathcal{M}^{\oplus}(\alpha, \beta ; \eta, \zeta, \boldsymbol{\tau}, \boldsymbol{\rho})=0 .
$$

In other words, the infimum is attained for the optimal state $\eta=y$ and adjoint state $\zeta=p$ with the optimal control $u=u_{d}-\frac{1}{\lambda} p$, and for the exact fluxes $\boldsymbol{\tau}=\nu \nabla y$ and $\boldsymbol{\rho}=\nu \nabla p$.

Proof. We have that

$$
\mathcal{M}^{\oplus}(\alpha, \beta ; y(u), p(u), \nu \nabla y(u), \nu \nabla p(u))=\frac{\alpha}{2}\left\|y(u)-y_{d}\right\|^{2},
$$

which is zero if we let $\alpha$ go to zero.

Finally, we repeat the results of Theorems 6 and Proposition 2 for the unconstrained case.

Theorem 9. Let $U_{a d}=L^{2}(\Omega)$. For any $\eta, \zeta \in V$, we obtain the following error majorant:

$$
\left\|u-v_{\zeta}\right\|_{1}^{2} \leq \mathcal{M}_{1}^{\oplus}(\alpha, \beta ; \eta, \zeta, \tau, \boldsymbol{\rho})
$$


with $\left\|u-v_{\zeta}\right\|_{1}^{2}=\|\| u-u_{d}+\frac{1}{\lambda} \zeta \mid \|_{1}^{2}$ and

$$
\begin{aligned}
\mathcal{M}_{1}^{\oplus}(\alpha, \beta ; & \eta, \zeta, \boldsymbol{\tau}, \boldsymbol{\rho})=\frac{\alpha}{2}\left\|\eta-y_{d}\right\|^{2}+\frac{(1+\alpha)(1+\beta) C_{F}^{2}}{2 \alpha \underline{\nu}^{2}}\|\boldsymbol{\tau}-\nu \nabla \eta\|^{2} \\
& +\frac{(1+\alpha)(1+\beta) C_{F}^{4}}{2 \alpha \beta \underline{\nu}^{2}}\left\|f+u_{d}-\frac{1}{\lambda} \zeta+\operatorname{div} \boldsymbol{\tau}\right\|^{2}-\int_{\Omega}\left(f+u_{d}-\frac{1}{\lambda} \zeta+\operatorname{div} \boldsymbol{\tau}\right) \zeta d \boldsymbol{x} \\
& -\int_{\Omega}(\boldsymbol{\tau}-\nu \nabla \eta) \cdot \nabla \zeta d \boldsymbol{x}+\frac{1}{\underline{\nu}}\left(C_{F}\left\|\eta-y_{d}+\operatorname{div} \boldsymbol{\rho}\right\|+\|\boldsymbol{\rho}-\nu \nabla \zeta\|\right) \\
& \times\left(\frac{C_{F}^{3}}{\lambda \underline{\nu}}\left\|\eta-y_{d}+\operatorname{div} \boldsymbol{\rho}\right\|+\frac{C_{F}^{2}}{\lambda \underline{\nu}}\|\boldsymbol{\rho}-\nu \nabla \zeta\|+C_{F}\left\|f+u_{d}-\frac{1}{\lambda} \zeta+\operatorname{div} \boldsymbol{\tau}\right\|+\|\boldsymbol{\tau}-\nu \nabla \eta\|\right) \\
& +\frac{3 \lambda}{2 C_{F}^{2}}\left(\|\boldsymbol{\tau}-\nu \nabla \eta\|+C_{F}\left\|f+u_{d}-\frac{1}{\lambda} \zeta+\operatorname{div} \boldsymbol{\tau}\right\|\right)^{2}
\end{aligned}
$$

for arbitrary $\tau, \boldsymbol{\rho} \in H(\operatorname{div}, \Omega)$ and $\alpha, \beta>0$.

Proof. Applying (34) in the unconstrained case as well as (10) and (39) finally leads to the estimate (44).

Proposition 4. Let $U_{a d}=L^{2}(\Omega)$. The majorant $\mathcal{M}_{1}^{\oplus}(\alpha, \beta ; \eta, \zeta, \boldsymbol{\tau}, \boldsymbol{\rho})$ defined in (44) attains the exact lower bound on the exact solution of the optimal control problem (1)-(2), or, equivalently, of the optimality system (6), (7) and (9), i.e.,

$$
\inf _{\substack{\eta, \zeta \in V, \boldsymbol{\tau}, \boldsymbol{\rho} \in H(\operatorname{div}, \Omega) \\ \alpha, \beta>0}} \mathcal{M}_{1}^{\oplus}(\alpha, \beta ; \eta, \zeta, \boldsymbol{\tau}, \boldsymbol{\rho})=0 .
$$

The infimum is attained for the optimal state $\eta=y$ and adjoint state $\zeta=p$ with the optimal control $u=u_{d}-\frac{1}{\lambda} p$, and for the exact fluxes $\boldsymbol{\tau}=\nu \nabla y$ and $\boldsymbol{\rho}=\nu \nabla p$.

Proof. We have that

$$
\mathcal{M}_{1}^{\oplus}(\alpha, \beta ; y(u), p(u), \nu \nabla y(u), \nu \nabla p(u))=\frac{\alpha}{2}\left\|y(u)-y_{d}\right\|^{2},
$$

which is zero if we let $\alpha$ go to zero.

\section{The Finite Element Discretization and the Preconditioned MinRes Solver}

In this section, we present the finite element discretization of the optimality system in order to derive approximations of the state, control and adjoint state. These approximations can be used for the computation of the majorants and minorants. We start with the unconstrained case.

The unconstrained case. Since the control can be eliminated from the optimality system by using (9), we only have to solve the system (6)-(7) for the state $y \in V$ and the adjoint state $p \in V$. For that, we approximate these unknown functions by finite element functions $y_{h}, p_{h} \in V_{h} \subset V$, where the finite element space $V_{h}$ is defined as

$$
V_{h}=\operatorname{span}\left\{\varphi_{1}, \ldots, \varphi_{n}\right\}
$$

with the standard nodal basis $\left\{\varphi_{i}(\boldsymbol{x})=\varphi_{i h}(\boldsymbol{x}): i=1,2, \ldots, n_{h}\right\}$ and $h$ denotes the discretization parameter (mesh size) such that $n=n_{h}=\operatorname{dim} V_{h}=O\left(h^{-d}\right)$. Using continuous, piecewise linear finite elements on triangles on a regular triangulation to construct the finite element subspace $V_{h}$ and its basis (see, e.g., [6]), yields the following linear system:

$$
\left(\begin{array}{cc}
M_{h} & -K_{h} \\
-K_{h} & -\lambda^{-1} M_{h}
\end{array}\right)\left(\begin{array}{c}
\underline{y}_{h} \\
\underline{p}_{h}
\end{array}\right)=\left(\begin{array}{c}
\underline{y}_{d} \\
-\left(\underline{f}^{+} \underline{u}_{d}\right)
\end{array}\right),
$$

which has to be solved with respect to the nodal parameter vectors $\underline{y}_{h}=\left(y_{i}\right)_{i=1, \ldots, n} \in \mathbb{R}^{n}$ and $\underline{p}_{h}=\left(p_{i}\right)_{i=1, \ldots, n} \in \mathbb{R}^{n}$ of the finite element approximations

$$
y_{h}(\boldsymbol{x})=\sum_{i=1}^{n} y_{i} \varphi_{i}(\boldsymbol{x}) \quad \text { and } \quad p_{h}(\boldsymbol{x})=\sum_{i=1}^{n} p_{i} \varphi_{i}(\boldsymbol{x})
$$


to the unknown functions $y(\boldsymbol{x})$ and $p(\boldsymbol{x})$. The matrices $M_{h}$ and $K_{h}$ correspond to the mass and stiffness matrices, respectively. Their entries are computed by the following formulas:

$$
M_{h}^{i j}=\int_{\Omega} \varphi_{i} \varphi_{j} d \boldsymbol{x}, \quad K_{h}^{i j}=\int_{\Omega} \nu \nabla \varphi_{i} \cdot \nabla \varphi_{j} d \boldsymbol{x}, \quad i, j=1, \ldots, n .
$$

The right hand sides are given by

$$
\underline{y}_{d}=\left[\int_{\Omega} y_{d} \varphi_{j} d \boldsymbol{x}\right]_{j=1, \ldots, n}, \quad \underline{u}_{d}=\left[\int_{\Omega} u_{d} \varphi_{j} d \boldsymbol{x}\right]_{j=1, \ldots, n} \quad \text { and } \quad \underline{f}=\left[\int_{\Omega} f \varphi_{j} d \boldsymbol{x}\right]_{j=1, \ldots, n} .
$$

The discretized optimality system (46) is a saddle point problem and can be solved by a preconditioned MINRES (minimal residual) method, see [28. Hence, it is crucial to construct preconditioners, which yield robust and fast convergence for the preconditioned MINRES method. In [37, the following preconditioner was constructed:

$$
\mathcal{P}=\left(\begin{array}{cc}
M_{h}+\sqrt{\lambda} K_{h} & 0 \\
0 & \frac{1}{\lambda} M_{h}+\frac{1}{\sqrt{\lambda}} K_{h}
\end{array}\right)
$$

leading to a robust convergence with respect to $h$ and $\lambda$ (as well as $\nu$ for our problem).

The constrained case. In the case of having inequality constraints imposed on the control, we can reformulate the variational inequality (8), which is equivalent to the projection formula

$$
u(\boldsymbol{x})=\mathbb{P}_{\left[u_{a}(\boldsymbol{x}), u_{b}(\boldsymbol{x})\right]}\left\{u_{d}(\boldsymbol{x})-\frac{1}{\lambda} p(\boldsymbol{x})\right\},
$$

by introducing an additional parameter $\mu \in L^{2}(\Omega)$, see, e.g., [13, 38]. The idea is to apply a primal-dual active set strategy in order to linearize the optimality system, see, e.g., [12]. The parameter $\mu$ is defined as $\mu=-\frac{1}{\lambda} p+u_{d}-u$ for the exact solution $u \in U_{\text {ad. }}$. Moreover, if a function $v \in U_{\text {ad }}$ satisfies the relations

$$
v(\boldsymbol{x})= \begin{cases}u_{a}(\boldsymbol{x}) & \text { if } v(\boldsymbol{x})+\mu(\boldsymbol{x})<u_{a}(\boldsymbol{x}), \\ u_{d}(\boldsymbol{x})-\frac{1}{\lambda} p(\boldsymbol{x}) & \text { if } v(\boldsymbol{x})+\mu(\boldsymbol{x}) \in\left[u_{a}(\boldsymbol{x}), u_{b}(\boldsymbol{x})\right], \\ u_{b}(\boldsymbol{x}) & \text { if } v(\boldsymbol{x})+\mu(\boldsymbol{x})>u_{b}(\boldsymbol{x}),\end{cases}
$$

then it is the optimal solution, since this means that $v$ satisfies the projection formula. In the following, we want to state the main steps of the primal-dual active set method for the optimal control problem (6)-(8). Given an iterate $\left(y_{k-1}, u_{k-1}, p_{k-1}, \mu_{k-1}\right)$, the active and inactive sets are determined as follows:

$$
\begin{gathered}
\mathcal{A}_{k}^{a}=\left\{\boldsymbol{x} \in \Omega: u_{k}(\boldsymbol{x})+\mu_{k}(\boldsymbol{x})<u_{a}(\boldsymbol{x})\right\}, \quad \mathcal{A}_{k}^{b}=\left\{\boldsymbol{x} \in \Omega: u_{k}(\boldsymbol{x})+\mu_{k}(\boldsymbol{x})>u_{b}(\boldsymbol{x})\right\}, \\
\mathcal{I}_{k}=\Omega \backslash\left(\mathcal{A}_{k}^{a} \cup \mathcal{A}_{k}^{b}\right) .
\end{gathered}
$$

If $\mathcal{A}_{k}^{a}=\mathcal{A}_{k-1}^{a}$ and $\mathcal{A}_{k}^{b}=\mathcal{A}_{k-1}^{b}$, then we have attained the optimal solution. Otherwise, the next iterate is the solution of the system

$$
\begin{aligned}
-\operatorname{div}\left(\nu(\boldsymbol{x}) \nabla y_{k}(\boldsymbol{x})\right) & =f(\boldsymbol{x})+u_{k}(\boldsymbol{x}), \quad \boldsymbol{x} \in \Omega, \quad y_{k}(\boldsymbol{x})=0, \boldsymbol{x} \in \Gamma, \\
-\operatorname{div}\left(\nu(\boldsymbol{x}) \nabla p_{k}(\boldsymbol{x})\right) & =y_{k}(\boldsymbol{x})-y_{d}(\boldsymbol{x}), \quad \boldsymbol{x} \in \Omega, \quad p_{k}(\boldsymbol{x})=0, \boldsymbol{x} \in \Gamma, \\
u_{k}(\boldsymbol{x})-u_{d}(\boldsymbol{x})+\lambda^{-1} \chi_{\mathcal{I}_{k}} p_{k}(\boldsymbol{x}) & =\chi_{\mathcal{A}_{k}^{a}} u_{a}(\boldsymbol{x})+\chi_{\mathcal{A}_{k}^{b}} u_{b}(\boldsymbol{x}), \quad \boldsymbol{x} \in \Omega,
\end{aligned}
$$

where $\chi$ denotes the characteristic function. We eliminate again the control $u_{k}$ from the system and obtain the following reduced optimality system written in its variational formulation:

$$
\begin{aligned}
\int_{\Omega} y_{k} w d \boldsymbol{x}-\int_{\Omega} \nu \nabla p_{k} \cdot \nabla w d \boldsymbol{x} & =\int_{\Omega} y_{d} w d \boldsymbol{x} \\
\int_{\Omega} \nu \nabla y_{k} \cdot \nabla w d \boldsymbol{x}+\lambda^{-1} \int_{\mathcal{I}_{k}} p_{k} w d \boldsymbol{x} & =\int_{\Omega}\left(f+u_{d}\right) w d \boldsymbol{x}+\int_{\mathcal{A}_{k}^{a}} u_{a} w d \boldsymbol{x}+\int_{\mathcal{A}_{k}^{b}} u_{b} w d \boldsymbol{x},
\end{aligned}
$$


for all $w, q \in V$. Now, discretizing the reduced optimality system by the finite element method with the space $V_{h} \subset V$ defined as in (45) leads to the linear system

$$
\left(\begin{array}{cc}
M_{h} & -K_{h} \\
-K_{h} & -\lambda^{-1} M_{h, \mathcal{I}_{k}}
\end{array}\right)\left(\begin{array}{l}
\underline{y}_{h, k} \\
\underline{p}_{h, k}
\end{array}\right)=\left(\begin{array}{c}
\underline{y}^{\underline{y}} d \\
-\left(\underline{f}+\underline{u}_{d}\right)-\underline{u}_{a}-\underline{u}_{b}
\end{array}\right),
$$

where the matrix $M_{h, \mathcal{I}_{k}}$ with the entries

$$
M_{h, \mathcal{I}_{k}}^{i j}=\int_{\mathcal{I}_{k}} \varphi_{i} \varphi_{j} d \boldsymbol{x}, \quad i, j=1, \ldots, n,
$$

and the vectors

$$
\underline{u}_{a}=\left[\int_{\mathcal{A}_{k}^{a}} u_{a} \varphi_{j} d \boldsymbol{x}\right]_{j=1, \ldots, n} \text { and } \underline{u}_{b}=\left[\int_{\mathcal{A}_{k}^{b}} u_{b} \varphi_{j} d \boldsymbol{x}\right]_{j=1, \ldots, n}
$$

have to be computed in every $k$-th iteration step of the level set method. In order to solve the linear system (49) by the preconditioned MINRES method, we need again a preconditioner, which provides fast convergence and is as robust as possible. Here, we refer to the preconditioner

$$
\mathcal{P}_{\mathcal{I}_{k}}=\left(\begin{array}{cc}
M_{h}+\sqrt{\lambda} K_{h} & 0 \\
0 & \frac{1}{\lambda} M_{h, \mathcal{I}_{k}}+\frac{1}{\sqrt{\lambda}} K_{h}
\end{array}\right),
$$

which was presented in [17]. For the preconditioner (50), it can be proven robustness with respect to the inactive set $\mathcal{I}_{k}$ and the mesh size $h$ (as well as with respect to the diffusion parameter $\nu$ in our case), but not with respect to the cost parameter $\lambda$ (the condition number scales like $1 / \sqrt{\lambda}$ ).

\section{Numerical Results}

In this section, we present and discuss first numerical results for the unconstrained case. The computational domain $\Omega=(0,1) \times(0,1)$ is uniformly decomposed into triangles, and standard continuous, piecewise linear finite elements are used for the discretization. In this case, the Friedrichs constant is $C_{F}=1 /(\sqrt{2} \pi)$.

The construction of $\eta, \zeta, \tau$ and $\rho$ is an important issue in order to obtain sharp guaranteed bounds from the majorants and minorants. Since $\eta$ and $\zeta$ are constructed by continuous, piecewise linear approximations, their gradients are only piecewise constant. Then, $\nabla \eta, \nabla \zeta \in L^{2}(\Omega)$, but $\nabla \eta, \nabla \zeta \notin H(\operatorname{div}, \Omega)$. Hence, a flux reconstruction is needed in order to obtain suitable fluxes $\boldsymbol{\tau}, \boldsymbol{\rho} \in H(\operatorname{div}, \Omega)$. A good reconstruction of the flux is an important and nontrivial topic. We can regularize the fluxes by a post-processing operator which maps the $L^{2}$-functions into $H(\operatorname{div}, \Omega)$, see 35. There are various techniques for realizing these post-processing steps such as, e.g., local post-processing by an elementwise averaging procedure or by using Raviart-Thomas elements, see [35, 25] and references, therein. In our numerical experiments, we use Raviart-Thomas elements of the lowest order, see, e.g., [29, 5, 36. We define the normal fluxes on interior edges $E_{m n}$ by

$$
\begin{aligned}
& \left.\left(\boldsymbol{\tau} \cdot n_{E_{m n}}\right)\right|_{E_{m n}}=\left(\left.\lambda_{m n}(\nabla \eta)\right|_{T_{m}}+\left.\left(1-\lambda_{m n}\right)(\nabla \eta)\right|_{T_{n}}\right) \cdot n_{E_{m n}}, \\
& \left.\left(\boldsymbol{\rho} \cdot n_{E_{m n}}\right)\right|_{E_{m n}}=\left(\left.\lambda_{m n}(\nabla \zeta)\right|_{T_{m}}+\left.\left(1-\lambda_{m n}\right)(\nabla \zeta)\right|_{T_{n}}\right) \cdot n_{E_{m n}},
\end{aligned}
$$

with $\lambda_{m n}=1 / 2$ due to uniform discretization. Here, $\left.(\nabla \eta)\right|_{T_{m}},\left.(\nabla \zeta)\right|_{T_{m}},\left.(\nabla \eta)\right|_{T_{n}}$ and $\left.(\nabla \zeta)\right|_{T_{n}}$ are constant vectors on two arbitrary, neighboring elements $T_{m}$ and $T_{n}$. On boundary edges, the only one existing flux is used. Hence, three normal fluxes are defined on the three sides of each element. Inside, we reconstruct the fluxes $\boldsymbol{\tau}$ and $\boldsymbol{\rho}$ by the standard lowest-order Raviart-Thomas $\left(\mathrm{RT}^{0}-\right)$ extension of normal fluxes with

$$
\begin{aligned}
\mathrm{RT}^{0}\left(\mathcal{T}_{h}\right):=\left\{\boldsymbol{\tau} \in\left(L^{2}(T)\right)^{2}: \forall T \in \mathcal{T}_{h} \quad \exists a, b, c \in \mathbb{R} \quad \forall \boldsymbol{x} \in T,\right. \\
\\
\left.\boldsymbol{\tau}(\boldsymbol{x})=(a, b)^{T}+c \boldsymbol{x} \text { and }[\boldsymbol{\tau}]_{E} \cdot n_{E}=0 \forall \text { interior edges } E\right\},
\end{aligned}
$$

where $[\boldsymbol{\tau}]_{E}$ denotes the jump of $\boldsymbol{\tau}$ across the edge $E$ shared by two neighboring elements on a triangulation $\mathcal{T}_{h}$. Altogether, the $\mathrm{RT}^{0}$-extension yields averaged fluxes from $H($ div, $\Omega)$, i.e.,

$$
\boldsymbol{\tau}=G_{\mathrm{RT}}(\nabla \eta), \quad \boldsymbol{\rho}=G_{\mathrm{RT}}(\nabla \zeta), \quad G_{\mathrm{RT}}: L^{2}(\Omega) \rightarrow H(\operatorname{div}, \Omega) .
$$


Results for functional a posteriori error estimates of parabolic time-periodic boundary value problems using the same discretization and flux reconstruction techniques can be found in [19.

In order to solve the saddle point systems (46), we use the AMLI preconditioner proposed by Kraus in [18 for an inexact realization of the block-diagonal preconditioner (48) in the MINRES method. The numerical results where computed on grids of different mesh sizes (from $8 \times 8$ to $256 \times 256)$. The preconditioned MINRES iteration was stopped after 8 iteration steps in all computations using the AMLI preconditioner with 4 inner iterations. The presented CPU times in seconds $t^{\mathrm{sec}}$ include the computational times for computing the majorants and minorants, which are very small in comparison to the computational times of the solver. All computations were performed on a laptop with Intel(R) Core(TM) i5-4308U CPU @ 2.80GHz.

In the numerical experiment, we consider the following given data: the desired state

$$
y_{d}(\boldsymbol{x})=\left(1+0.04 \pi^{4}\right) \sin \left(x_{1} \pi\right) \sin \left(x_{2} \pi\right),
$$

the desired control $u_{d}(\boldsymbol{x})=0$ and the source term $f(\boldsymbol{x})=0$. We choose the parameter $\nu=1$ and the cost parameter $\lambda=0.01$. The exact state and control are known and given by

$$
y(\boldsymbol{x})=\sin \left(x_{1} \pi\right) \sin \left(x_{2} \pi\right) \quad \text { and } \quad u(\boldsymbol{x})=2 \pi^{2} \sin \left(x_{1} \pi\right) \sin \left(x_{2} \pi\right),
$$

respectively. Hence, the exact value of the cost functional is given by $\mathcal{J}(y(u), u)=2.385$. Table 1 presents the CPU times in seconds $t^{\mathrm{sec}}$, the majorants (37) and minorants (38) as well as the efficiency indices

$$
\begin{aligned}
I_{\mathrm{eff}}^{\oplus} & =\frac{\mathcal{J}^{\oplus}(\alpha, \beta ; \eta, \zeta, \boldsymbol{\tau})}{\mathcal{J}(y(u), u)}, & I_{\mathrm{eff}}^{\ominus} & =\frac{\mathcal{J}(y(u), u)}{\mathcal{J}^{\ominus}(\eta, \zeta, \boldsymbol{\tau}, \boldsymbol{\rho})}, \\
I_{\mathrm{eff}}^{\oplus / \ominus} & =\frac{\mathcal{J}^{\oplus}(\alpha, \beta ; \eta, \zeta, \boldsymbol{\tau})}{\mathcal{J}^{\ominus}(\eta, \zeta, \boldsymbol{\tau}, \boldsymbol{\rho})}, & I_{\mathrm{eff}}^{\mathcal{M}_{1}} & =\sqrt{\frac{\mathcal{M}_{1}^{\oplus}(\alpha, \beta ; \eta, \zeta, \boldsymbol{\tau})}{\|\| u-v_{\zeta} \|_{1}^{2}}}
\end{aligned}
$$

obtained on grids of different mesh sizes. The parameters $\alpha$ and $\beta$ are chosen by a proper minimization of the majorant $\mathcal{J}^{\oplus}$ with respect to $\alpha$ and $\beta$, see [25]. In Table 2], the individual parts of the majorants and minorants are presented. They are denoted as follows:

$$
\begin{aligned}
& \mathcal{R}_{1}^{\eta}=\left\|\operatorname{div} \boldsymbol{\tau}-\frac{1}{\lambda} \zeta\right\|, \quad \mathcal{R}_{1}^{\zeta}=\left\|\eta-y_{d}+\operatorname{div} \boldsymbol{\rho}\right\|, \quad \mathcal{R}_{2}^{\eta}=\|\boldsymbol{\tau}-\nabla \eta\|, \quad \mathcal{R}_{2}^{\zeta}=\|\boldsymbol{\rho}-\nabla \zeta\|, \\
& \mathcal{R}_{3}^{\eta}=\left\|\eta-y_{d}\right\|, \quad \mathcal{R}_{3}^{\zeta}=\|\zeta\|, \quad \mathcal{R}_{4}=\int_{\Omega}\left(\operatorname{div} \boldsymbol{\tau}-\frac{1}{\lambda} \zeta\right) \zeta d \boldsymbol{x}, \quad \mathcal{R}_{5}=\int_{\Omega}(\boldsymbol{\tau}-\nu \nabla \eta) \cdot \nabla \zeta d \boldsymbol{x} .
\end{aligned}
$$

\begin{tabular}{|c|ccccccc|}
\hline grid & $t^{\mathrm{sec}}$ & $\mathcal{J}^{\ominus}$ & $\mathcal{J}^{\oplus}$ & $I_{\text {eff }}^{\oplus}$ & $I_{\text {eff }}^{\ominus}$ & $I_{\text {eff }}^{\oplus / \ominus}$ & $I_{\text {eff }}^{\mathcal{M}_{1}}$ \\
\hline $8 \times 8$ & 0.006 & 2.248 & 2.438 & 1.022 & 1.061 & 1.085 & 2.369 \\
$16 \times 16$ & 0.012 & 2.351 & 2.431 & 1.019 & 1.015 & 1.034 & 1.953 \\
$32 \times 32$ & 0.045 & 2.376 & 2.412 & 1.011 & 1.004 & 1.015 & 1.751 \\
$64 \times 64$ & 0.179 & 2.383 & 2.399 & 1.006 & 1.001 & 1.007 & 1.656 \\
$128 \times 128$ & 0.784 & 2.384 & 2.392 & 1.003 & 1.000 & 1.003 & 1.610 \\
$256 \times 256$ & 3.246 & 2.385 & 2.389 & 1.002 & 1.000 & 1.002 & 1.587 \\
\hline
\end{tabular}

TABLE 1. Efficiency of the minorants and majorants.

In both tables, we observe the efficiency of the AMLI preconditioned MINRES method presented in [18, 20]. The computational times increase with a factor of four. Moreover, one can see that $\mathcal{R}_{1}^{\eta}$ and $\mathcal{R}_{1}^{\zeta}$ reduce as a factor of two, and $\mathcal{R}_{2}^{\eta}$ and $\mathcal{R}_{2}^{\zeta}$ as a factor of four showing the efficiency of the applied flux reconstruction. However, one could consider other flux reconstruction techniques in terms of improving the efficiency indices such as using higher order Raviart-Thomas elements, see 25]. Altogether we can observe that the majorants and minorants provide good estimates for the value of the cost functional and can be used in order to compute guaranteed estimates for the discretization error in the combined norms as it is discussed and proved in this paper. 


\begin{tabular}{|c|cccccccc|}
\hline grid & $\mathcal{R}_{1}^{\eta}$ & $\mathcal{R}_{1}^{\zeta}$ & $\mathcal{R}_{2}^{\eta}$ & $\mathcal{R}_{2}^{\zeta}$ & $\mathcal{R}_{3}^{\eta}$ & $\mathcal{R}_{3}^{\zeta}$ & $\mathcal{R}_{4}$ & $\mathcal{R}_{5}$ \\
\hline $8 \times 8$ & 1.146 & 0.235 & 0.053 & 0.011 & 1.928 & 0.094 & -0.013 & 0.001 \\
$16 \times 16$ & 0.606 & 0.121 & 0.016 & 0.003 & 1.943 & 0.098 & -0.004 & 0.000 \\
$32 \times 32$ & 0.307 & 0.061 & 0.004 & 0.001 & 1.947 & 0.098 & -0.001 & 0.000 \\
$64 \times 64$ & 0.154 & 0.030 & 0.001 & 0.000 & 1.948 & 0.099 & -0.000 & 0.000 \\
$128 \times 128$ & 0.077 & 0.015 & 0.000 & 0.000 & 1.948 & 0.099 & -0.000 & 0.000 \\
$256 \times 256$ & 0.039 & 0.008 & 0.000 & 0.000 & 1.948 & 0.099 & -0.000 & 0.000 \\
\hline
\end{tabular}

TABLE 2. The indiviudal parts of the minorants and majorants.

\section{Conclusions}

This work was devoted to the derivation of guaranteed and fully computable lower bounds (minorants) for cost functionals of distributed elliptic optimal control problems in order to close the gap of the already existing results on upper bounds for these cost functionals, see [7]. An important result of this work was to prove that the discretization error in the state and the control can be computed by the difference between majorant and minorant of the cost functional. Altogether we derive a fully computable upper bound for the discretization error in the state and the control, which can be, in principle, used as object of direct minimization. However, the aim of this work was not to present an algorithm for this minimization, but to derive first results on minorants for cost functionals of distributed elliptic optimal control problems with control constraints as well as present first numerical tests for the theoretical results derived.

\section{ACKNOWLEDGMENTS}

The author gratefully acknowledges the financial support by the Austrian Academy of Sciences, and thanks U. Langer, S. Repin and G. Wachsmuth for the fruitful discussions and valuable comments regarding a posteriori error estimation.

\section{REFERENCES}

[1] R. Becker, M. Brafck, D. Meidner, R. Rannacher, and B. Vexler, Adaptive finite element methods for PDE-constrained optimal control problems, in Reactive flows, diffusion and transport, Springer, 2007, pp. $177-205$.

[2] R. Becker, H. Kapp, And R. Rannacher, Adaptive finite element methods for optimal control of partial differential equations: Basic concept, SIAM Journal on Control and Optimization, 39 (2000), pp. $113-132$.

[3] R. Becker And R. Rannacher, An optimal control approach to a posteriori error estimation in finite element methods, Acta Numerica 2001, 10 (2001), pp. 1-102.

[4] A. Borzì And V. Schulz, Computational Optimization of Systems Governed by Partial Differential Equations, SIAM, Philadelphia, 2012.

[5] F. Brezzi and M. Fortin, Mixed and hybrid finite element methods, vol. 15 of Springer Series in Computational Mathematics, Springer-Verlag, New York, 1991.

[6] P. G. Ciarlet, The Finite Element Method for Elliptic Problems, Studies in Mathematics and its Applications 4, North-Holland, Amsterdam, 1978. Republished by SIAM in 2002.

[7] A. Gaevskaya, R. H. W. Hoppe, and S. Repin, A posteriori estimates for cost functionals of optimal control problems, Numerical Mathematics and Advanced Applications, Proceedings of the ENUMATH 2005, (2006), pp. 308-316.

[8] — - Functional approach to a posteriori error estimation for elliptic optimal control problems with distributed control, Journal of Mathematical Sciences, 144 (2007), pp. 4535-4547.

[9] A. Günther and M. Hinze, A posteriori error control of a state constrained elliptic control problem, Journal of Numerical Mathematics, 16 (2008), pp. 307-322.

[10] M. Hintermüller and R. H. W. Hoppe, Goal-oriented adaptivity in control constrained optimal control of partial differential equations, SIAM Journal on Control and Optimization, 47 (2008), pp. 1721-1743.

[11] M. Hintermüller, R. H. W. Hoppe, Y. Iliash, And M. Kieweg, An a posteriori error analysis of adaptive finite element methods for distributed elliptic control problems with control constraints, ESAIM: Control, Optimisation and Calculus of Variations, 14 (2008), pp. 540-560.

[12] M. Hintermüller, K. Ito, And K. Kunisch, The primal-dual active set strategy as a semismooth Newton method, SIAM J. Optim., 13 (2002), pp. 865-888.

[13] M. Hinze, R. Pinnau, M. Ulbrich, and S. Ulbrich, Optimization with PDE constraints, Mathematical Modelling: Theory and Applications 23, Springer, Berlin, 2009. 
[14] R. H. W. Hoppe And M. Kieweg, Adaptive finite element methods for mixed control-state constrained optimal control problems for elliptic boundary value problems, Computational Optimization and Applications, 46 (2010), pp. 511-533.

[15] K. Kohls, A. Rösch, And K. G. Siebert, A posteriori error estimators for control constrained optimal control problems, in Constrained Optimization and Optimal Control for Partial Differential Equations, Springer, 2012, pp. 431-443.

[16] K. Kohls, A. Rösch, And K. G. Siebert, A posteriori error analysis of optimal control problems with control constraints, SIAM Journal on Control and Optimization, 52 (2014), pp. 1832-1861.

[17] M. Kollmann, Efficient Iterative Solvers for Saddle Point Systems arising in PDE-constrained Optimization Problems with Inequality Constraints, PhD thesis, JKU Linz, 2013.

[18] J. Kraus, Additive Schur complement approximation and application to multilevel preconditioning, SIAM J. Sci. Comput., 34 (2012), pp. A2872-A2895.

[19] U. Langer, S. RePin, And M. Wolfmayr, Functional a posteriori error estimates for parabolic time-periodic boundary value problems, Comput. Methods Appl. Math., (2015).

[20] U. Langer and M. Wolfmayr, Multiharmonic finite element analysis of a time-periodic parabolic optimal control problem, J. Numer. Math., 21 (2013), pp. 265-300.

[21] G. Leugering, P. Benner, S. Engell, A. Griewank, H. Harbrecht, M. Hinze, R. Rannacher, and S. UlbRich, Trends in PDE Constrained Optimization, Birkhäuser, Basel, 2015.

[22] G. Leugering, S. Engell, A. Griewank, M. Hinze, R. Rannacher, V. Schulz, M. Ulbrich, and S. Ulbrich, Constrained Optimization and Optimal Control for Partial Differential Equations, Birkhäuser, Basel, 2012.

[23] J. L. Lions, Optimal Control on Systems Governed by Partial Differential Equations, Springer, BerlinHeidelberg-New York, 1971.

[24] W. Liu And N. Yan, A posteriori error estimates for distributed convex optimal control problems, Advances in Computational Mathematics, 15 (2001), pp. 285-309.

[25] O. Mali, P. Neittaanmäki, and S. Repin, Accuracy Verification Methods. Theory and Algorithms, Computational Methods in Applied Sciences 32, Springer, Netherlands, 2014.

[26] S. G. Mikhlin, Variational methods in mathematical physics, Pergamon Press Oxford, 1964.

[27] P. Neittaanmäki and S. Repin, Reliable Methods for Computer Simulation: Error Control and Posteriori Estimates, vol. 33, Elsevier, 2004

[28] C. C. Paige and M. A. Saunders, Solution of sparse indefinite systems of linear equations, SIAM J. Numer. Anal., 12 (1975), pp. 617-629.

[29] P. A. Raviart and J. M. Thomas, A mixed finite element method for 2-nd order elliptic problems, Mathematical Aspects of Finite Element Methods, Lect. Notes Math. 606, (1977), pp. 292-315.

[30] S. Repin, A posteriori error estimates for approximate solutions to variational problems with strongly convex functionals, Problems of Mathematical Analysis, 17 (1997), pp. 199-226. In Russian. Translated in J. Math. Sci. 97(4):4311-4328, 1999.

[31] — A posteriori error estimation for nonlinear variational problems by duality theory, Zapiski Nauchn, Semin, 249 (1997), pp. 244-255. In Russian. Translated in J. Math. Sci. 99(1):927-935, 2000.

[32] - A unified approach to a posteriori error estimation based on duality error majorants, Mathematics and Computers in Simulation, 50 (1999), pp. 305-321.

[33] - Estimates of deviations from exact solutions of elliptic variational inequalities, Zapiski Nauchn, Semin, 271 (2000), pp. 188-203. In Russian. Translated in J. Math. Sci. 115(6):2811-2819, 2003.

[34] - A posteriori error estimation for variational problems with uniformly convex functionals, Math. Comput., 69 (2000), pp. 481-500.

[35] — A Posteriori Estimates for Partial Differential Equations, Radon Series on Computational and Applied Mathematics 4, Walter de Gruyter, Berlin, 2008.

[36] J. E. Roberts and J. M. Thomas, Mixed and hybrid methods, Handbook of numerical analysis, 2 (1991), pp. 523-639.

[37] J. Schöberl and W. Zulehner, Symmetric indefinite preconditioners for saddle point problems with applications to PDE-constrained optimization problems, SIAM J. Matrix Anal. Appl., 29 (2007), pp. 752-773.

[38] F. Tröltzsch, Optimal Control of Partial Differential Equations. Theory, Methods and Applications, Graduate Studies in Mathematics 112, AMS, Providence, RI, 2010.

[39] B. Vexler and W. Wollner, Adaptive finite elements for elliptic optimization problems with control constraints, SIAM Journal on Control and Optimization, 47 (2008), pp. 509-534.

(M. Wolfmayr) Johann Radon Institute for Computational and Applied Mathematics, Austrian Academy of Sciences, Altenbergerstrasse 69, 4040 Linz, Austria

E-mail address: monika.wolfmayr@ricam.oeaw.ac.at 